\title{
Acute spinal cord injury associated with multilevel pediatric idiopathic intervertebral disc calcification: case report
}

\author{
Sandi K. Lam, MD, MBA, ${ }^{1}$ Christian M. Niedzwecki, DO, ${ }^{2}$ Bradley Daniels, BS, ${ }^{1}$ Rory R. Mayer, MD, ${ }^{1}$ \\ Mili M. Vakharia, FNP-C, ${ }^{2}$ and Andrew Jea, MD1 \\ 'Division of Pediatric Neurosurgery, Texas Children's Hospital, and Department of Neurosurgery, Baylor College of Medicine; and \\ 2Division of Pediatric Rehabilitation, Texas Children's Hospital, and Department of Physical Medicine and Rehabilitation, Baylor \\ College of Medicine, Houston, Texas
}

\begin{abstract}
Pediatric idiopathic intervertebral disc calcification (PIIVDC) is a rare condition; most cases are reported to be selflimited with conservative management. In this study, we describe a case of PIIVDC presenting with acute incomplete spinal cord injury with Brown-Séquard-plus syndrome that was treated with surgery and demonstrate the subsequent rehabilitation time course.
\end{abstract}

http://thejns.org/doi/abs/10.3171/2015.6.PEDS15214

KEY WORDS spinal cord injury; spine; Brown-Séquard-plus syndrome; pediatric; intervertebral disc calcification

$\mathrm{P}$ EDIATRIC idiopathic intervertebral disc calcification (PIIVDC) is an uncommon condition typically affecting children ages $5-12$ years. ${ }^{2,5}$ It is rarely associated with a calcified nucleus pulposus herniation into the spinal canal to the extent that it causes neurological compromise. ${ }^{9}$ Our case represents a rare presentation of PIIVDC with Brown-Séquard-plus syndrome (BSPS). Brown-Séquard-type injuries occur in 1\%-4\% of all spinal cord injuries (SCIs) per year. ${ }^{13}$ BSPS is characterized by motor loss on one side of the body and pain and temperature loss on the opposite side of the body, plus additional neurological deficits, including bladder dysfunction.

\section{Case Report}

History and Examination

An athletic 12-year-old girl presented with acute right lower-extremity numbness and weakness that was described as the right leg "giving out" and falling while she was singing in a choir concert. Prior to this event, she had been followed by her primary care practitioner for a 2-week history of constant midthoracic back pain believed to be musculoskeletal in nature. She had no history of trauma or fever. She had difficulties with urinary incontinence for at least 6 months prior to presentation.

The patient's physical examination revealed right lower-extremity weakness (Grade 4/5 hip flexion/extension/ abduction/adduction, knee flexion/extension; Grade 2/5 ankle dorsiflexion; Grade 0/5 big toe extensor; and Grade 0/5 plantar flexion); impaired temperature (hot and cold) sensation in her left lower extremity; and impaired vibratory sensation in her right lower extremity. Great toe proprioception was intact bilaterally. The patient's rectal tone and sensation were intact. Her patellar reflexes were Grade $3+$ bilaterally. She had 4-beat ankle clonus on the right and 2-beat ankle clonus on the left. Her Babinski reflexes were noted to be downgoing bilaterally. Bilateral upper extremities were normal. Her SCI was determined to be American Spinal Injury Association (ASIA) Grade D.

Imaging

MRI and CT scanning revealed a calcified lesion within the anterior portion of the spinal canal extending from C-7 to the inferior endplate of T-3. This mass originated from

ABBREVIATIONS ASIA = American Spinal Injury Association; BSPS = Brown-Séquard-plus syndrome; $C R P=$ C-reactive protein; $D$ DISH = diffuse idiopathic skeletal hyperostosis; ESR = erythrocyte sedimentation rate; OPLL = ossification of the posterior longitudinal ligament; PIIVDC = pediatric idiopathic intervertebral disc calcification; SCI = spinal cord injury.

SUBMITTED April 8, 2015. ACCEPTED June 18, 2015.

INCLUDE WHEN CITING Published online October 16, 2015; DOI: 10.3171/2015.6.PEDS15214. 
the T1-2 disc space. There were multilevel calcifications within the intervertebral disc spaces of C7-T1, T1-2, T23, and T3-4 (Fig. 1). The posterior longitudinal ligament did not have diffuse thickening and did not contain calcifications. Inflammatory markers C-reactive protein (CRP) and erythrocyte sedimentation rate (ESR) were within normal limits. The calcified disc herniation occupied half of the spinal canal, causing cord compression most prominently at the level of T-2. T2-weighted MRI showed a hyperintense signal in the cord parenchyma, suggesting cord edema (Fig. 2). The imaging findings and clinical presentation were typical of the rare entity of PIIVDC, and the diagnosis was made.

\section{Operation}

Given the acute neurological deficit associated with spinal cord compression, it was recommended that the patient undergo surgical decompression and stabilization. A T1-2 laminectomy, right transpedicular decompression, and C5-T4 posterior instrumented fusion were performed.

Pathological examination of material removed from the spinal canal via transpedicular decompressions showed fibrocartilaginous tissue with reactive/regenerative changes consistent with intervertebral disc.

\section{Rehabilitation}

Postoperatively, the patient received 2 weeks of inpatient rehabilitation to treat residual impairments in sensory, neuromuscular, bladder, and bowel function. Her distal right lower-extremity muscle groups improved postoperatively within 24 hours to Grade 3/5 motor strength. Based on results of her neuromuscular examination on admission to the inpatient rehabilitation unit, her injury was classified as a T-6 ASIA Grade D incomplete paraplegia. She was unable to complete a single-leg stance on either leg and required 25\% assistance to stand and ambulate. Because of her right knee hyperextension and foot drop with each step, a right articulated ankle-foot orthosis was used. Notably, her activities of daily living were more impaired than her mobility. She required over $75 \%$ assistance for lower-extremity dressing, bathing, and toileting. With
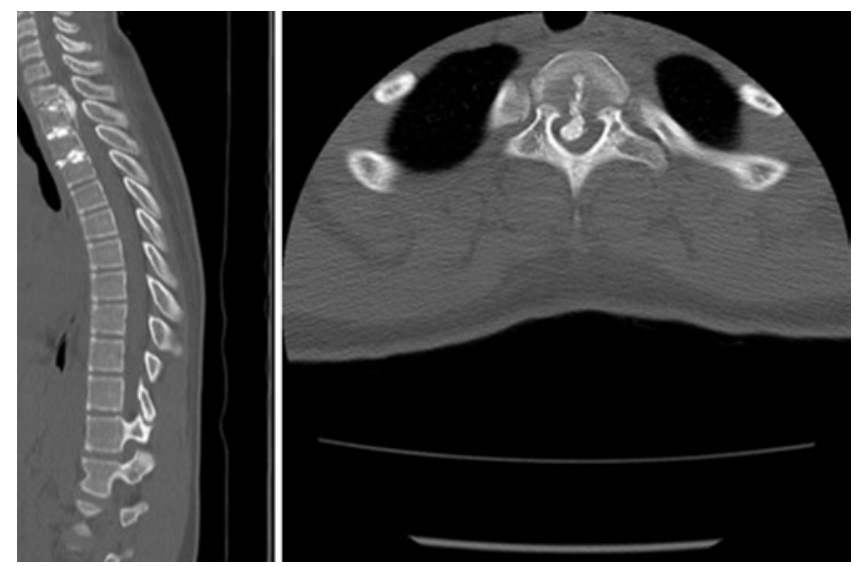

FIG. 1. Left: Sagittal CT scan in bone windows showing calcified disc spaces and associated calcified extrusion. Right: Axial CT scan at the level of T-2 showing a lesion extension from the intervertebral disc space and occupying a significant portion of the spinal canal.

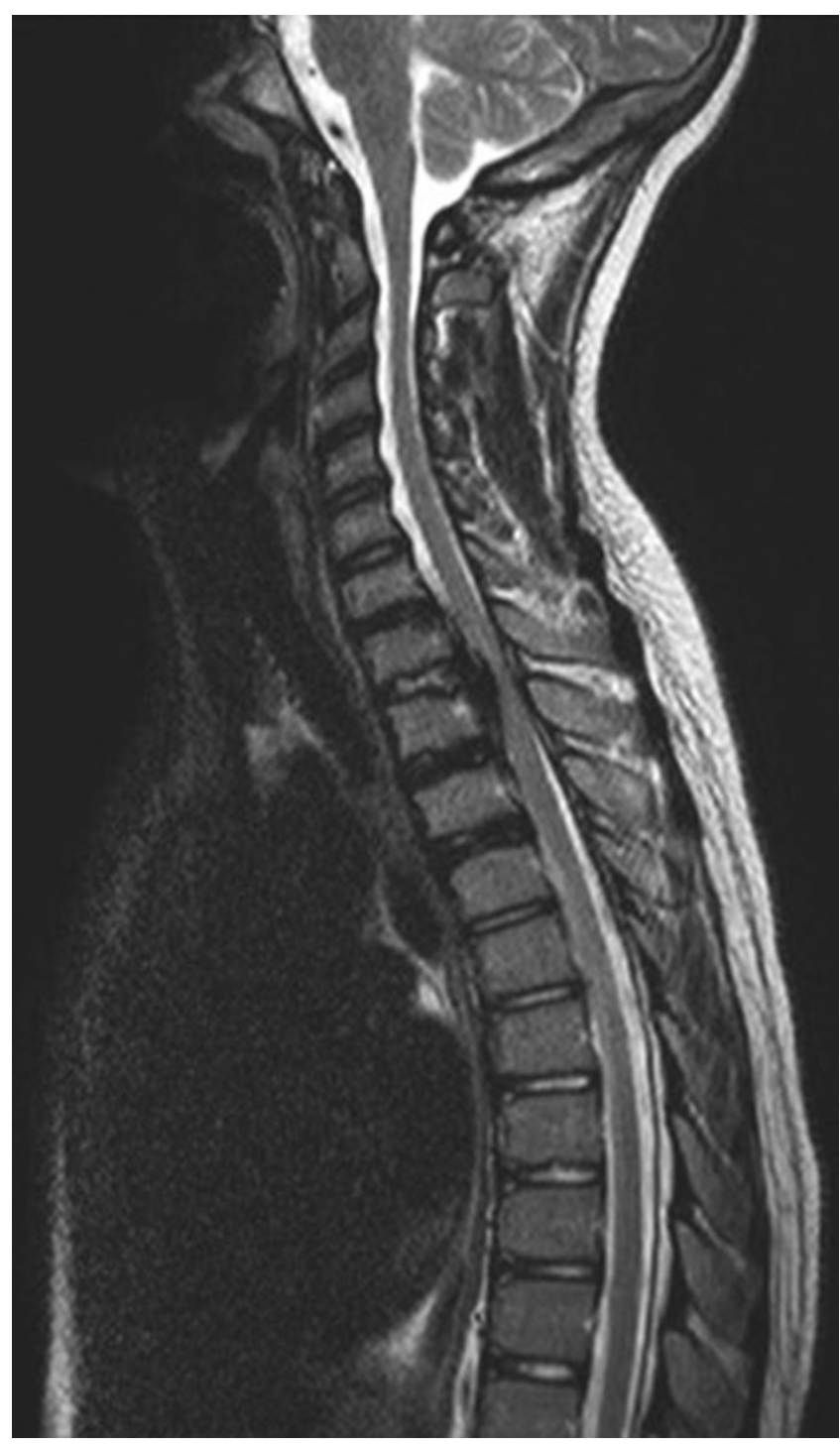

FIG. 2. Sagittal T2-weighted MR image demonstating spinal cord compression, most severe at the level of T1-2.

regard to bladder function, she responded well to timed voiding and did not require intermittent catheterization. Her bowel management required oral medications and no suppositories. Her initial functional independence score (via WeeFIM) was 86 out of a possible 126 points.

On discharge from inpatient rehabilitation, she had attained a modified level of independence in her ability to ambulate in a controlled environment and in her activities of daily living, with significant improvement in her injury classification to an L-3 ASIA Grade D incomplete paraplegia. Her discharge functional independence score (via WeeFIM) was 111 of 126 points. She was discharged to home with a right articulated ankle-foot orthosis and durable medical equipment items such as a tub transfer chair and a reacher.

\section{Follow-Up}

At her 3-month follow-up, the patient's neuromuscular deficits and functional independence had fully recovered 
(ASIA Grade E), although her bladder function remained under investigation. A CT scan at that time showed complete resolution of the intraspinal calcific lesion and reconstitution of the spinal canal diameter (Fig. 3). At her 6-month follow-up, the patient had regained bladder continence and had resumed active pursuits such as competitive dance.

\section{Discussion}

The overall incidence of PIIVDC is low, with approximately 400 cases reported since $1924 .{ }^{21}$ PIIVDC tends to affect more males than females, and presentation is typically from 5 to 12 years of age. ${ }^{2}$ Although principal symptoms include pain in the region of calcification (71.9\%), sensorimotor disturbance (20.3\%), low-grade fever $(13.8 \%)$, torticollis $(23.4 \%),{ }^{21}$ decreased range of motion, stiffness, and paraspinal muscle spasm with or without torticollis, ${ }^{2}$ some children do not report any pain symptoms and, at times, the radiological diagnosis may be an incidental finding. ${ }^{16}$ In fact, a review of 65 cases between 1990 and 2010 reported that $15.4 \%$ of patients had an asymptomatic presentation..$^{21}$

While the etiology of PIIVDC is poorly understood, the natural process of intervertebral disc maturation might provide insight into the increased susceptibility to PIIVDC in 5- to 12-year-old children. During this time span, the intervertebral disc nutritional supply structure is moving away from a microvascular-based system to a mature osmotic nonvascular system. These changes place the intervertebral discs in a particularly vulnerable state to ischemia and necrosis. This view not only would tend to support recent studies that propose that a viral-induced vasculitis disrupts the blood supply within intervertebral discs and bodies, leading to dystrophic calcification development, but also would support the findings that approximately $50 \%$ of PIIVDC cases are associated with leukocytosis and elevated inflammatory markers such as ESR and CRP. ${ }^{4-7}$ Some patients also have been noted to present with elevated protein in the CSF. ${ }^{2}$ Logically, trauma may serve as an initiator for the inflammatory response; however, most cases reported have no history of trauma. ${ }^{4}$

PIIVDC without sensorimotor disturbance is self-limiting and can be managed effectively with medications (e.g. analgesics and muscle relaxants) and immobilization of the affected spinal segment. Over time, the calcified lesion is resorbed. ${ }^{6,21}$ In the rare instance when the calcified nucleus pulposus herniates into the spinal canal to the extent of causing neurological compromise, ${ }^{9}$ the decision to treat surgically compared with conservatively depends on the severity of neurological sequelae. Operative management typically involves discectomy and interbody fusion with or without corpectomy., ${ }^{4,5,9,22}$ Medical treatment is more common; $, 1,6,7,16,17$ only a handful of reported cases are managed with surgical intervention..$^{4,5,9,10,12}$

In our case, the pathology specimen obtained at surgery showed fibrocartilaginous tissue with reactive/regenerative changes consistent with intervertebral disc. Reports with descriptions of the pathology specimen are not plentiful in the literature. While some groups have described an inflammatory response with histiocytes and

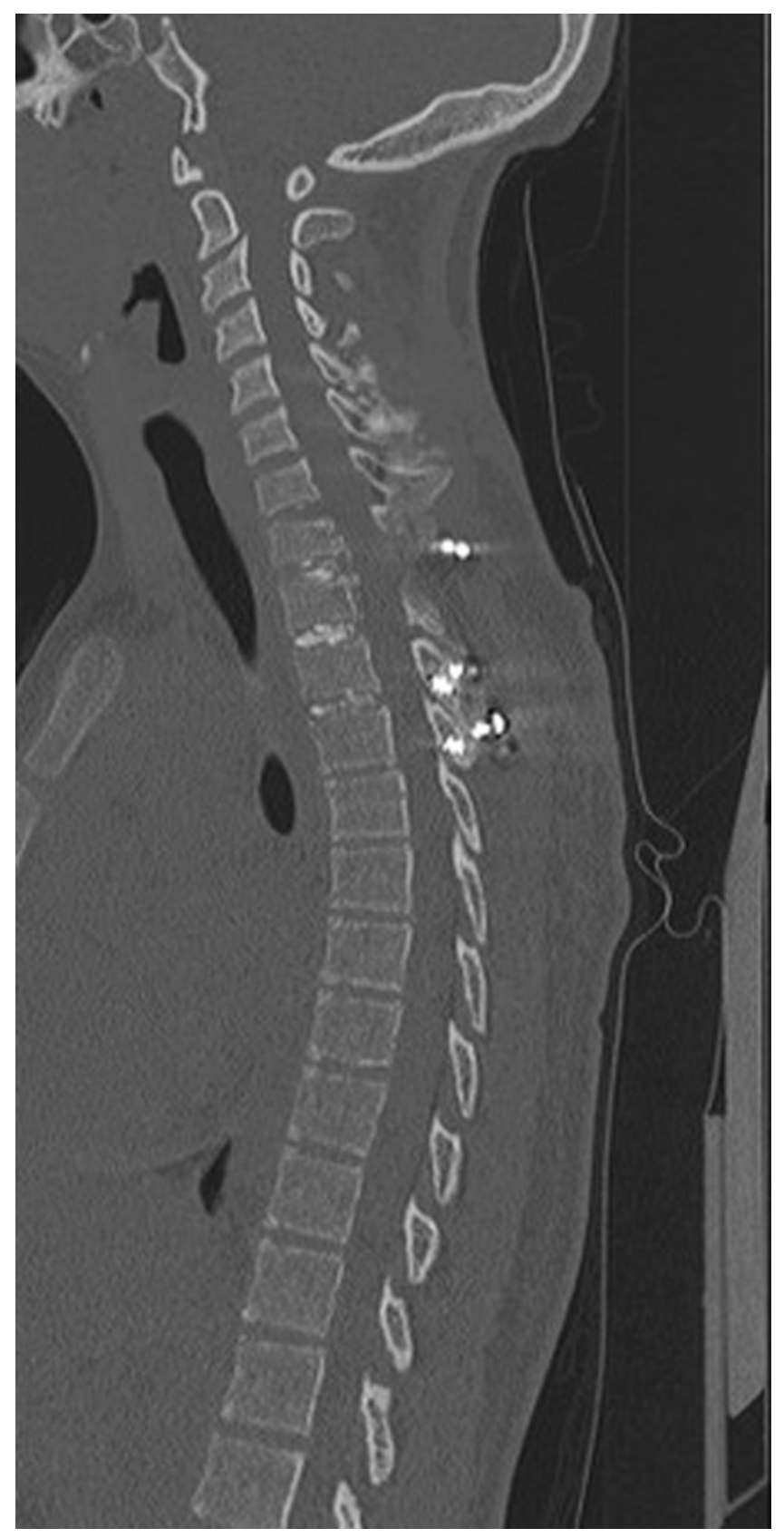

FIG. 3. Sagittal CT scan in bone windows at 3 months after surgery showing complete resolution of the intraspinal calcified mass. Calcified material in the intervertebral disc spaces C7-T1, T1-2, T2-3, and T3-4 exists without herniation or extrusion.

foreign body-type giant cells in the removed disc material, ${ }^{14,18}$ others have found neither inflammatory nor reactive changes in the excised disc material. ${ }^{5,20}$ Our current patient appeared to fall within this spectrum of disease.

It is useful to distinguish between PIIVDC and other ossifying disease entities of the spinal column. Diffuse idiopathic skeletal hyperostosis (DISH) is a syndrome of ossification of the soft tissue and ligaments, typically at the ventral aspect of the cervical and thoracic spine at the anterior longitudinal ligament. Most patients are asymptomatic, though dysphagia can occur when bony overgrowth 
of the anterior longitudinal ligament leads to compression of the esophagus. DISH is characterized as an adult disease and has an incidence of $15 \%-30 \%$ in patients over 65 years. ${ }^{19}$ DISH and ossification of the posterior longitudinal ligament (OPLL) can occur together, though DISH is more common in Caucasian populations, while OPLL is predominantly described in Asian populations. OPLL occurs in adults typically over 40 years of age. The posterior longitudinal ligament exists at the dorsal aspects of the vertebral bodies and intervertebral discs. It extends from the occiput to the sacrum, though OPLL is most often diagnosed in the cervical spine. As the posterior longitudinal ligament hypertrophies and ossifies, narrowing of the spinal canal diameter can cause ventral cord compression, cervical myelopathy, and/or SCI. The dura also can be involved in the ossification process. Early radiological signs of OPLL include an enlarged posterior longitudinal ligament with small areas of bone contained within this ligament. With progression, these areas form large bony plaques ventral to the spinal cord..$^{19}$ Intervertebral discs are not affected.

Our patient's workup exemplified classic features of PIIVDC, occurring in a patient younger than 20 years old, with localized pain symptoms and characteristic radiological features. The imaging findings included calcification in the intervertebral disc spaces without evidence of other degenerative processes or abnormalities in the posterior longitudinal ligament. This calcified disc material contiguously extruded into the spinal canal. Our case falls into the rare subset of cases with severe progressive neurological deficit presenting with BSPS from PIIVDC, which required surgical treatment. There were no constitutional signs (i.e., no fever) or laboratory findings suggestive of inflammation (i.e., ESR and CRP were normal), and, in agreement with existing literature, pain in the region affected by calcification and sensorimotor disturbance ${ }^{21}$ was a major feature of this patient's disease.

In this case, we see multiple variances from previously reported cases, including thoracic level of lesion, multiple levels of disc calcification, severe neurological deficits requiring surgical treatment, and posterior surgical approach. Neurological symptoms are almost always associated with protrusion of disc material into the spinal canal, which has been identified in $38 \%$ of cases. ${ }^{11}$ Thoracic lesions remain neurologically asymptomatic more often than cervical lesions, with nearly one in three patients with radiological thoracic disease presenting without symptoms. ${ }^{15}$ Though low cervical is the most common location for the calcification (64.6\% of cases, with a peak frequency at C6-7), involvement of upper thoracic intervertebral discs, as in our case, is not unseen (26.2\%). ${ }^{15}$ Calcification of discs at multiple levels spanning both cervical and thoracic segments is rare, reported in just $10 \%$ of cases. ${ }^{4}$

The need for surgical decompression in the present case makes our patient part of a rare subgroup of patients in whom acute neurological impairment warranted surgical management. Rather than the typical anterior approach for corpectomy/discectomy and anterior interbody fusion, we performed a posterior approach for decompression and instrumented fusion. An anterior approach was briefly considered, but a corpectomy at this challenging T1-2 level would have presented a higher level of morbidity, with obstruction by the manubrium, proximity to the upper lung tissue, and proximity to the great vessels. Laminectomy and transpedicular decompression were followed by posterior instrumented fusion to avoid postoperative kyphosis and eliminate micromotion (i.e., exacerbation of the extrusion). Thus, we deemed the posterior approach to be the safest possible choice in this situation to relieve cord compression and to stabilize the spine, especially with the understanding that the anterior pathology was expected to resorb.

To further support the surgical approach taken with the presentation of BSPS, Kohno et al. showed that surgical treatment of incomplete SCI, including Brown-Séquard syndrome, has been shown to halt the progressive loss of neurological function. ${ }^{8}$ The prognosis for significant motor recovery in BSPS is good,,$^{13}$ and symptoms can continue to improve for up to 2 years. The common pattern of recovery is that of ipsilateral proximal extensors before distal flexors, and increased strength in the sensory deficit-affected limb before the contralateral limb. Functional gait will typically return within 1-6 months of injury. ${ }^{13}$ Our patient achieved full functional recovery by 6 months after surgery.

Improvement in symptoms and pathology occurred slowly in weeks to months after surgery. This length of recovery is comparable to conservatively managed cases with lesser neurological deficit. ${ }^{1,3,5,6,15}$ The success of the patient's treatment course likely was attributable to urgent decompression of her spinal cord, surgical stabilization of the spinal segment affected by intervertebral calcification to prevent further extrusion, and collaboration with the Physical Medicine and Rehabilitation team.

The ultimate goal in the management of PIIVDC will be prevention of disc herniation and neurological compromise. Short of that goal, it is important to aim to reasonably predict the clinical outcomes for patients with a given severity of disease and a proposed treatment plan.

\section{Conclusions}

PIIVDC is a rare entity typically affecting the pediatric population. While it is generally treated by conservative management, with resorption of herniated calcified lesions over time, surgical intervention is indicated in cases that involve spinal cord compression with acute neurological compromise.

\section{References}

1. Bajard X, Renault F, Benharrats T, Mary P, Madi F, Vialle R: Intervertebral disc calcification with neurological symptoms in children: report of conservative treatment in two cases. Childs Nerv Syst 26:973-978, 2010

2. Chu J, Wang T, Pei S, Yin Z: Surgical treatment for idiopathic intervertebral disc calcification in a child: case report and review of the literature. Childs Nerv Syst 31:123-127, 2015

3. Cronqvist S, Mortensson W: Protrusion of calcified cervical discs into the spinal canal in children. A report of two cases. Neuroradiology 9:223-225, 1975

4. Dönmez H, Mavili E, Ikizceli T, Koç RK: Pediatric intervertebral disc calcification. Diagn Interv Radiol 14:225-227, 2008 
5. Gerlach R, Zimmermann M, Kellermann S, Lietz R, Raabe A, Seifert V: Intervertebral disc calcification in childhooda case report and review of the literature. Acta Neurochir (Wien) 143:89-93, 2001

6. Girodias JB, Azouz EM, Marton D: Intervertebral disk space calcification. A report of 51 children with a review of the literature. Pediatr Radiol 21:541-546, 1991

7. Ho C, Chang S, Fulkerson D, Smith J: Children presenting with calcified disc herniation: a self-limiting process. J Radiol Case Rep 6:11-19, 2012

8. Kohno M, Takahashi H, Yamakawa K, Ide K, Segawa H: Postoperative prognosis of Brown-Séquard-type myelopathy in patients with cervical lesions. Surg Neurol 51:241-246, 1999

9. Lernout C, Haas H, Rubio A, Griffet J: Pediatric intervertebral disk calcification in childhood: three case reports and review of literature. Childs Nerv Syst 25:1019-1023, 2009

10. MacCartee CC Jr, Griffin PP, Byrd EB: Ruptured calcified thoracic disc in a child. Report of a case. J Bone Joint Surg Am 54:1272-1274, 1972

11. Mahlfeld K, Kayser R, Grasshoff H: Permanent thoracic myelopathy resulting from herniation of a calcified intervertebral disc in a child. J Pediatr Orthop B 11:6-9, 2002

12. Mainzer F: Herniation of the nucleus pulposus. A rare complication of intervertebral-disk calcification in children. Radiology 107:167-170, 1973

13. McKinley W, Santos K, Meade M, Brooke K: Incidence and outcomes of spinal cord injury clinical syndromes. J Spinal Cord Med 30:215-224, 2007

14. Oga M, Terada K, Kikuchi N, Oda Y, Sugioka Y: Herniation of calcified cervical intervertebral disc causes dissociated motor loss in a child. Spine (Phila Pa 1976) 18:2347-2350, 1993

15. Peck FC Jr: A calcified thoracic intervertebral disk with herniation and spinal cord compression in a child; case report. J Neurosurg 14:105-109, 1957

16. Sagiuchi T, Iida H, Tachibana S, Utsuki S, Tanaka R, Fujii $\mathrm{K}$ : Idiopathic spinal cord herniation associated with calcified thoracic disc extrusion-case report. Neurol Med Chir (Tokyo) 43:364-368, 2003
17. Sieroń D, Gruszczyńska K, Machnikowska-Sokołowska M, Olczak Z, Knap D, Baron J: Intervertebral disc calcification in children: Case description and review of relevant literature. Pol J Radiol 78:78-80, 2013

18. Smith RA, Vohman MD, Dimon JH III, Averett JE Jr, Milsap JH Jr: Calcified cervical intervertebral discs in children. Report of three cases. J Neurosurg 46:233-238, 1977

19. Smith ZA, Buchanan CC, Raphael D, Khoo LT: Ossification of the posterior longitudinal ligament: pathogenesis, management, and current surgical approaches. A review. Neurosurg Focus 30(3):E10, 2011

20. Swick HM: Calcification of intervertebral dises in childhood. J Pediatr 86:364-369, 1975

21. Tsutsumi S, Yasumoto Y, Ito M: Idiopathic intervertebral disk calcification in childhood: a case report and review of literature. Childs Nerv Syst 27:1045-1051, 2011

22. Yang HS, Chen DY, Yuan W, Yang LL, Tsai N, Lin QS: Paresis associated with aconuresis caused by intervertebral disc calcification at C7-T1: a case report and review of the literature. Spine (Phila Pa 1976) 35:E434-E439, 2010

\section{Disclosure}

The authors report no conflicts of interest concerning the materials or methods used in this study or the findings specified in this paper.

\section{Author Contributions}

Conception and design: Lam, Niedzwecki. Acquisition of data: Lam, Niedzwecki, Daniels. Analysis and interpretation of data: Mayer, Jea. Drafting the article: Lam, Daniels. Critically revising the article: Lam, Niedzwecki, Mayer, Vakharia, Jea. Reviewed submitted version of manuscript: Lam, Mayer. Study supervision: Lam, Jea.

\section{Correspondence}

Sandi Lam, Division of Pediatric Neurosurgery, Texas Children's Hospital, 6701 Fannin St., CCC Ste. 1230, Houston, TX 77030. email: sklam@texaschildrens.org. 